Cell and Organ Transplantology. 2018; 6(1):80-85 doi:10.22494/cot.v6i1.83

\title{
The effects of different time of melatonin administration on differentiation and functional status of the brown adipocytes in vivo
}

\author{
Kalmukova O. O., Dzerzhinsky M. E. \\ Educational and Scientific Centre "Institute of Biology", Taras Shevchenko National University of Kyiv, Kyiv, Ukraine \\ e-mail: olesiakalmukova28@gmail.com
}

\section{ABSTRACT}

Recently, numerous studies have indicated that melatonin, the hormone of pineal gland, affects the processes of differentiation of many cells, including preadipocytes into brown, beige and white adipocytes. Therefore, it is important to study the possibilities of using melatonin as a factor for the differentiation of preadipocytes to control the adipose tissue functional activity in the treatment of various diseases, including obesity.

THE AIM of the study was to evaluate the morphology and functional status of brown adipose tissue at the different time of melatonin administration.

MATERIALS AND METHODS. Outbred rats were divided into 3 experimental groups: control, administration of melatonin 1 hour after light-on ZT01 (Zeitgeber time), administration of melatonin 1 hour before light-off ZT11. Melatonin was administered by gavage daily for 7 weeks at a standard exposure to day-night light (12:12).

RESULTS. The application of melatonin affects the morphology and functional state of brown adipocytes independently of the administration time: the nuclear-cytoplasmic ratio increases due to an increase in the area of the nucleus against the unchanging cell area. The number of lipid drops increases in each adipocyte, but their size is decreased. The effect of different time of melatonin administration was manifested in the increase of brown adipose tissue weight, in the terms of evening administrations.

CONCLUSION. The effects of different times of melatonin administration at the cytological level are manifested almost identically in the brown adipocytes functional state in all experimental groups. However, at the organism level in terms of evening administrations, an increase in the relative mass of brown adipose tissue is observed, but the size of brown adipocytes do not change, which may indicate the activation of the differentiation of brown adipocytes from the progenitor cells.

KEY WORDS: brown adipocytes; cell differentiation; melatonin; chronobiology

Melatonin is a pineal gland hormone, which, besides the main function of regulating the circadian rhythm of the body, has many additional ones: immunomodulatory, antioxidant, anti-apoptotic, regulation of energy metabolism and others [1]. The realization of various functions of melatonin is carried out through the involvement of many mechanisms:

- $\quad$ integration of the circadian rhythm of the whole body: with the involvement of the subtle interaction of the hypothalamic suprachiasmatic nucleus (endogenous driver of the daily rhythm) and synchronized melatonin secretion by the epiphysis [2];

- direct elimination of free radicals: melatonin and its direct metabolites (cyclic hydroxymelatonin, N-acetyl-N-formyl-methoxykinuramine, $\mathrm{N}$-acetylmethoxykinuramine) provide reduc- tion of oxidative stress in a series of sequential dehydrogenation reactions, the formation of stable products with free radicals and increase donor-acceptor properties for a determined ordered electron flow [3];

- the other functions (immunomodulatory, antioxidant, anti-apoptic, thermoregulatory, anti-age, neurotrophic, etc. [4]) realized by the activation of melatonin receptors, which are present in almost all the cells of the body $[5,6]$, including stem cells of different origin.

Melatonin receptors are represented by two types of membrane receptors that are conjugated to G-proteins (MT1, MT2) [7, 8], and one type of nuclear orphan receptor of retinoic acid ROR superfamily $[9,10]$. 
Surface receptors have been found in many types of stem cells - neural $[11,12,13,14]$, mesenchymal (bone marrow-derived [15, 16], adiposederived [17], from cord blood [18]), embryonic [19], induced pluripotent [20], stem cells from the dental pulp [21]. Presence of melatonin receptors testifies to the possibility of the melatonin effect on proliferation, differentiation and functional activity of these cells, and in the future on the repair and recovery of tissues and the body [22, 23].

Due to the recent discovery of functionally active brown adipose tissue (BAT) in adults [24, 25], active research on the regulation mechanisms of its metabolic status is ongoing. This tissue may serve as a potential target for the treatment of metabolic disorders that may occur in the obesity, in the decreased insulin sensitivity (because adipose tissue is insulin-sensitive) and in the dyslipidemia [26, 27]. At present, different approaches for the modulation of BAT physiology are proposed:

- $\quad$ catecholamines - normally increase the heat-producing properties of BAT after activation by the cold of the hypothalamic thermoregulation centre and stimulation of the sympathetic nervous system, but they have a number of side effects $[28,29]$;

- $\quad$ irisin - myokine, which is secreted by myocytes during exercise and stimulates the mitochondrial biogenesis in both muscle and adipose tissue, which causes the appearance of classical brown adipocytes and beige adipocytes in white adipose tissue [30, 31];

- melatonin - not only increases the number of mitochondria in brown adipocytes, but also contributes to the increase in the number of BAT. Previously, it was hypothesized that such an effect of melatonin was mediated by the influence on the hypothalamic suprachiasmatic nucleus and subsequent activation of the sympathetic nervous system. A good example of this can be a seasonal rhythm of the BAT mass growth in species that enter the winter hibernation (brown bears, rodents and others) [32]. However, later it was shown that melatonin receptors are present in brown adipocytes, which makes melatonin an interesting agent for regulation the activity of these cells, as well as preadipocytes [33, 34]);

- cell therapy and tissue engineering - involves transplantation of brown adipocytes (for example, differentiated from adiposederived progenitors by transcription factors BMP7, PRDM16 or by adenoviral gene transfection), into the interscapular region to increase the mass of functionally active BAT in humans $[35,36,37]$, which may be useful in the treatment of obesity and metabolic syndrome [26, 27, 38]. Successful transplantation of such cells was carried out in mice and rats, but their effectiveness requires further investigation [39, 40]. In addition, synthetic matrices and scaffolds must be used for the successful engraftment of large transplants and to avoid post-transplantation necrosis [41, 42]

Physiology of BAT is easily regulated by melatonin, which not only increases its mass, but also increases the metabolic activity of brown adipocytes. Thus, melatonin is a non-toxic substance of natural origin, which is a highly conserved molecule that has biological effects in almost all organisms starting from antioxidant function in bacteria, and has no side effects [43, 44], can serve as a new approach to BAT activating [38]. Except influence through the hypothalamus, melatonin stimulates its membrane receptors on brown adipocytes [45] and may induce to the expression of thermogenin (UCP1), a protein that breaks the respiratory chain in mitochondria and provides non-shivering thermogenesis. Melatonin also induces PPARg expression (peroxisome proliferator-activated receptors gamma) and its co-activator PGC1a - key factors for the differentiation of new brown adipocytes and mitochondrial biogenesis [46]. A confirmation of this is a series of experiments in vitro on the culture of embryonic fibroblasts, which were directly differentiated into preadipocytes [47]. However, there are also opposite effects under melatonin treatment. Using the culture of human mesenchymal stem cells, it was shown a decrease in adipogenesis by inhibiting GSK-3 $\beta$ glycogen-synthase kinase [48], enhancing osteogenesis by increasing Runx2 expression and decreasing PPARg [49]. In the culture of embryonic fibroblasts differentiated into preadipocytes, inhibition of the transcription factor $\mathrm{C} / \mathrm{EBPb}$, which activates expression of PPARg, was shown [50]. Such ambiguous data obtained on cell cultures requires further in vivo studies with a detailed description of changes in the morphology of brown adipocytes and taking into account the relative weight of the BAT.

An additional interesting issue is the varying sensitivity of the organism to melatonin over the course of the day, as manifested by the differences in the membrane and nuclear receptors expression in cells [51, 52]. One of the topical issues in chronopharmacology is the choice of the effective time of drug administration to increase the useful effects and reduce the side effects $[53,54,55]$. Therefore, the aim of the study was to evaluate the morphology and functional status of brown adipose tissue at different time of melatonin administration.

\section{MATERIALS AND METHODS}

All experiments on animals are performed in compliance with the international principles of the European Convention for the Protection of Vertebrate Animals used for Experimental and other Scientific Purposes (Strasbourg, 1986), Article 26 of the Law of Ukraine «On the Protection of Animals from Cruelty» (No. 3447-IV, dated February 21, 2006), as well as all norms of bioethics, biological safety and general ethical principles of experiments on animals adopted by the First National Congress of Ukraine on Bioethics (September, 2001).

In our study, we used white outbred rats weighing 130-140 g, which were kept with a constant temperature regime under standard vivarium conditions and a diet with ad libitum access to water and food. As an external synchronizer of time, artificial lighting was used. The daily rhythm (Zeitgeber time - ZT) consisted of 12 hours of light and 12 hours of darkness with light switching on at 7 am (ZTO0) and off at 7 pm (ZT12). Rats were randomly divided into three groups of 7 animals in each: 1) control group; 2 ) the melatonin administration in 1 hour after the light turn on $-M$ ZT01; 3) the administration of melatonin 1 hour before light off - M ZT11 (Fig. 1). Melatonin (Alcon Biosciences, USA) was administered by gavage daily for 7 weeks at a dose of $30 \mathrm{mg} / \mathrm{kg} /$ day.

The calculation of water and feed consumption was carried out daily at 10:00 and calculated the relative daily water consumption $(\mathrm{ml} /$ day/ $\mathrm{kg}$ body mass) and food (kcal/day/kg body mass) for each animal. Body mass was measured once per week and calculated the weight gain of the body. On the last day of the experiment, the animals were decapitated, and then the brown adipose tissue was isolated from the interscapular region and weighed for the calculation of the relative mass.

Control

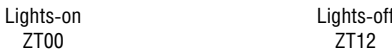

(1)

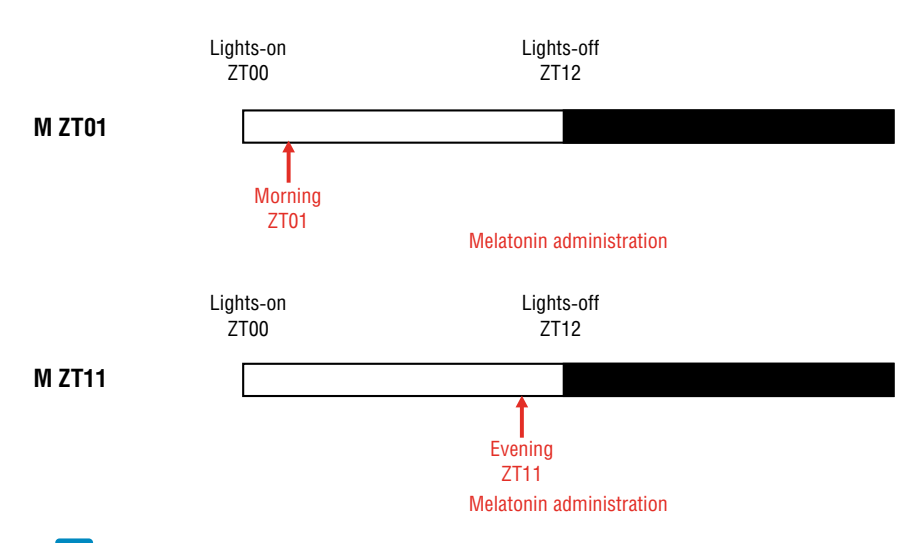

Fig. 1. Schemes of melatonin administration in experimental groups of rats. 
Histological examination was performed to characterize the morphology and functional status of brown adipose tissue. Fragments of brown adipose tissue in the size of $1 \times 1 \mathrm{~cm}$ were fixed in $4 \%$ of paraformaldehyde in $0.1 \mathrm{M}$ phosphate buffer for 72 hours, after which they were dehydrated and embedded into paraffin according to a standard procedure. From the paraffin blocks, $5 \mu \mathrm{m}$ sections were performed and stained with Bemer's hematoxylin and eosin. Further examination of sections was performed using a light microscope BX41 (Olympus, Japan). Microphotographs were taken using the DP20 (Olympus, Japan) digital camera and the QuickPHOTO MICRO software (Promicra, Czech Republic).

The cross-sectional area of the nucleus, cells and lipid droplets, nuclear-cytoplasmic ratio (NCR), the number of lipid inclusions per one cell and the optical density of the tissue were used as criteria for assessing the morphology and functional status of brown adipocytes. All parameters were measured using the ImageJ software (National Institutes of Heath, USA).

Statistical data analysis was performed using the Statistica 6.0 (StatSoft, USA) and Microsoft Excel 2010 software (Microsoft, USA). The distribution of values was estimated using Shapiro-Wilk W-test. Since the deviation of these values distribution of from the normality was minor to evaluate the differences between the values we used Student's t-test. The differences with probability of the null hypothesis $p<0.05$ were considered significant. The obtained results are presented as the mean \pm standard error of mean.

\section{RESULTS AND DISCUSSION}

By the morphology (Fig. 2), brown adipocytes differ from the white adipocytes by central location of the oval nucleus (in contrast to the peripheral arrangement of the flattened nucleus of white adipocytes); polygonal form of cells (white adipocytes are rounded), multilocular localization of numerous lipid droplets (in white adipocytes there is one large drop), large number of mitochondria and smaller linear cell sizes.

After the melatonin administration, the localization and form of the nucleus do not change, but the colour becomes light, that is, less basophilic. Cell morphology remains polygonal with a bright eosinophilic cytoplasm (Fig. 3).

According to the morphometric analysis, melatonin application caused the increase of brown adipocytes nucleus area by $46 \%$ in the group M ZT01 and by $60 \%$ in the M ZT11 group, and the evening administration induces these changes by $10 \%$ more in comparison with the morning one (Fig. 4). The cross-section area of the cell under the melatonin impact did not significantly change. Nuclear-cytoplasmic ratios after the melatonin action increased significantly in both schemes of administration - in M ZT01 group by $53 \%$, in M ZT11 group by 68 $\%$, respectively. The difference between melatonin-treated groups was greater in M ZT11 group by $10 \%$ compared with morning administration. This indicates an increase in the functional activity of brown adipocytes by stimulating synthetic processes in the nucleus, which may be due to the expression of a number of genes, including thermogenin [56].

At standard histological tissue processing, lipids, which are deposited in the adipocytes in the form of inclusions, are washed out during the dehydration of the tissue. Therefore, the places of their localization in the cytoplasm of the cell after staining with eosin remain uncoloured. At a smaller magnification of the lens, it is possible to estimate the overall brightness, which depends directly on the amount of lipids in the tissue: the tissue looks optically darker after the impact of melatonin (Fig. 5).

The data of the morphometric analysis confirm this: in the group M ZT01, the optical density of BAT (scale in standard units: 100 - black, 0 - white) increases by $29 \%$, while in M ZT11 group - by $21 \%$ (Fig. 6). This result is observed because the number of lipid droplets increases for each adipocyte: in M ZT01 group by $70 \%$ and in M ZT11 group by $60 \%$, with a decrease in their cross-sectional area - in M ZT01 group by $50 \%$ and in M ZT11 by $45 \%$. Since the optical density of BAT increases and more lipid droplets with diminished size appear there, under the condition of an unchanging area of adipocyte, according to literature data $[57,58]$, this may be an indirect indication of the growth of the number of mitochondria. This is because a greater number of mitochondria provides significant splitting of triacylglycerides, which leads to a gradual decrease in the size of lipid droplets. These data for reducing the size of lipid droplets are consistent with the data obtained on the culture of embryonic fibroblasts cells of 3T3-L1 mice after the addition of melatonin to the culture medium [47]. In that experiment, the authors also showed increased expression of PPARg.

Relative weight of BAT after melatonin administration increased significantly: in M ZT01 group by $20 \%$ and in M ZT11 group by $56 \%$, with a significant difference between melatonin-treated groups, by $30 \%$ higher in M ZT11 group compared to M ZT01. However, the effect on the
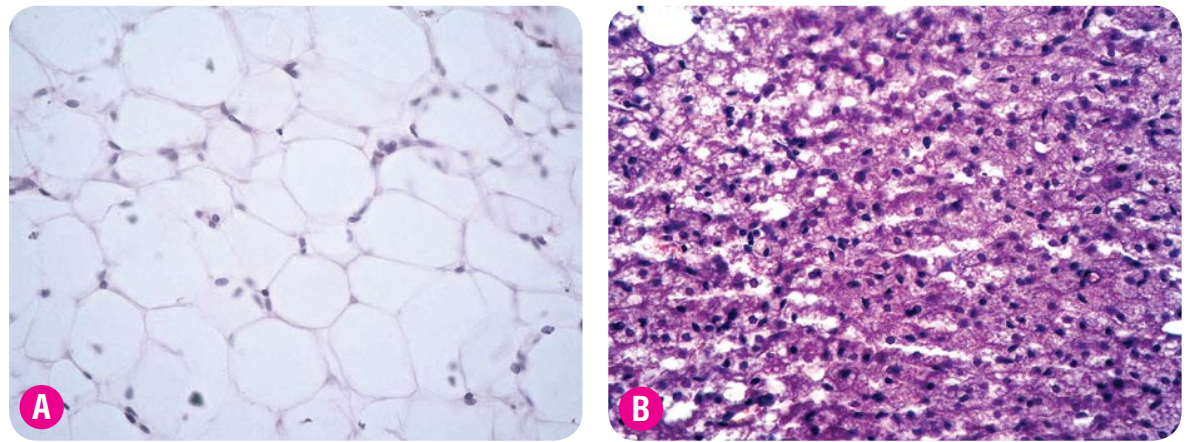

$<$ sections: A - white, B - brown;
hematoxylin-eosin staining; oc. x10, ob. x40.

Fig. 3 Microphotographs of rats' brown adipose tissue sections: A - control B - group M ZT01, C - group M ZT11; hematoxylin-eosin staining; oc. x10, ob. x100.
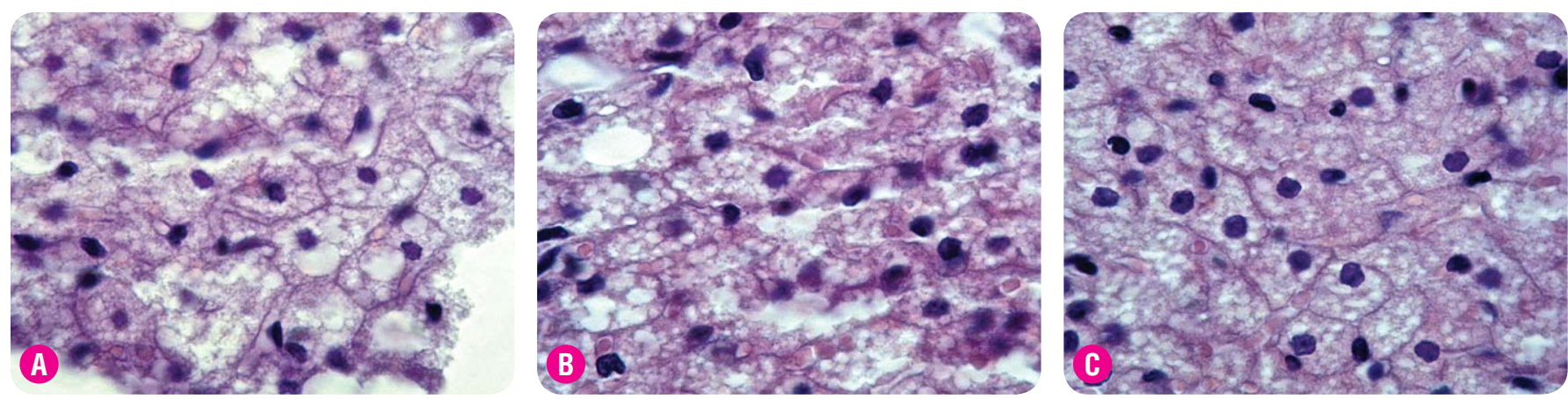
growth of the total mass was absent (Table 1). Such changes were not due to increased food consumption, but due to the stimulation of preadipocytes differentiation by melatonin in the direction of brown adipocytes, since adipocyte hypertrophy was not observed (the linear sizes of brown adipocytes did not change).
The difference in the effect of melatonin in different time of administration is due to the involvement of not only melatonin receptors on adipocytes, but also indirect signalling pathways through the inclusion of the hypothalamic nuclei and the peripheral nervous system.
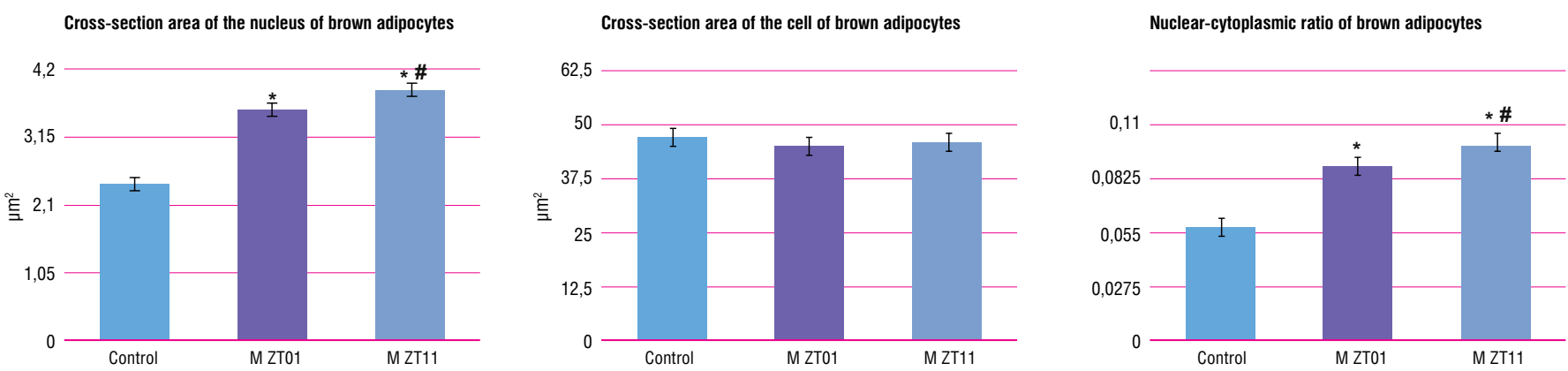

A Fig. 4. The morphometric analysis data of the cross-sectional area of the nucleus, cells and nuclear-cytoplasmic ratio of rats' brown adipocytes. Notes: * - a significant difference between the control and experimental groups, $p \leq 0.05$; \# - a significant difference between the groups M ZT01 and M ZT11, $p \leq 0.05$.
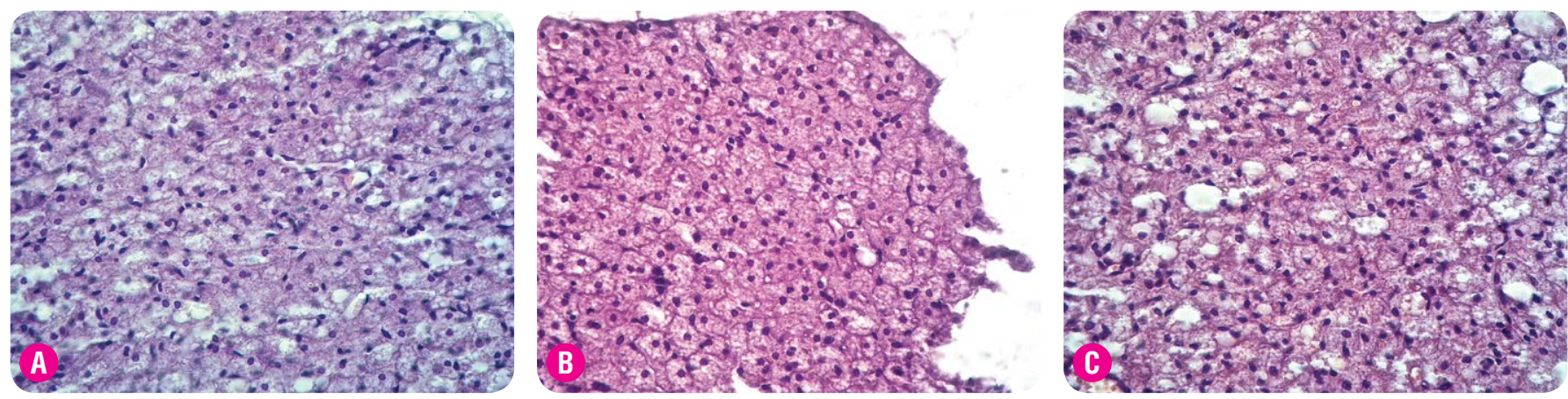

A Fig. 5 Microphotographs of rats' brown adipose tissue sections: A - control group, B - group M ZT01, C - group M ZT11; hematoxylin-eosin staining; oc. x10, ob. x40.
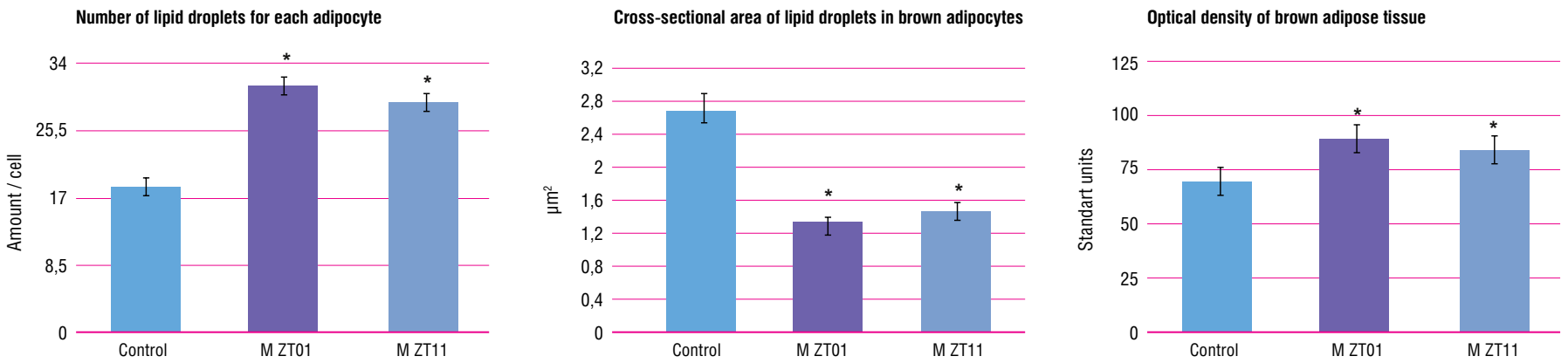

Fig. 6. The morphometric analysis data of the number, cross-sectional area of the lipid droplets in brown adipocytes and optical density of rats' BAT.

Notes: * - a significant difference between the control group and the experimental groups, $p \leq 0.05$; \# - a significant difference between the groups M ZTO1 and $M Z T 11, p \leq 0.05$.

Table 1. General characteristics of changes in mass of brown adipose tissue, body weight and food intake.

\begin{tabular}{|l|c|c|c|}
\hline \multicolumn{1}{|c|}{ PARAMETER } & CONTROL GROUP & GROUP M ZT01 & GROUP M ZT11 \\
\hline The relative weight of brown adipose tissue, $\%$ & $0.068 \pm 0.005$ & $0.082 \pm 0.011^{*}$ & $0.106 \pm 0.011^{*}$ \# \\
\hline Weight gain, $\%$ & $195 \pm 23$ & $236 \pm 16$ & $207 \pm 23$ \\
\hline Food consumption, kcal/g body mass per day & $0.247 \pm 0.002$ & $0.258 \pm 0.005$ & $0.245 \pm 0.003$ \\
\hline Water consumption, kcal/g body mass per day & $38.9 \pm 0.8$ & $39.3 \pm 0.9$ & $39.3 \pm 0.6$ \\
\hline
\end{tabular}




\section{CONCLUSION}

According to the morphofunctional analysis, melatonin enhances the adipogenesis of brown adipose tissue and activates the work of brown adipocytes, which manifests itself in changing the relative weight of this tissue and the number of lipid droplets in the cell.

This effect depends on the administration time. The effective mode of melatonin administration to activate the lipolysis of brown adipose tissue is evening (one hour before the lights off) because the area of the cross section of the brown adipocytes nucleus, the nuclear-cytoplasmic ratio and the relative weight of the brown adipose tissue increase compared to the morning (one hour after the light is turned on) administration.

The effects of different modes of administration of melatonin at the cytological level are almost identical in the functional state of brown adipocytes, but there is an increase in the relative mass of brown adipose tissue at the body level, at evening introductions.

\section{REFERENCES}

1. Hardeland R, Cardinali D, Srinivasan V, et al. Melatonin—A pleiotropic, orchestrating regulator molecule. Prog Neurobiol. 2011; 93(3):350-384.

2. Pfeffer $M$, Korf $H$, Wicht $H$. Synchronizing effects of melatonin on diurnal and circadian rhythms Gen Comp Endocrin. 2017; 258:215-221.

3. Reina M, Martínez A. A new free radical scavenging cascade involving melatonin and three of its metabolites (3OHM, AFMK and AMK). Comput Theoret Chem. 2018; 1123:111-118.

4. Acuña-Castroviejo D, Escames G, Venegas C, et al. Extrapineal melatonin: sources, regulation, and potential functions. Cell Mol Life Sci. 2014; 71(16):2997-3025

5. Ekmekcioglu C. Melatonin receptors in humans: biological role and clinical relevance. Biomed Pharmacother. 2006; 60(3):97-108.

6. Slominski RM, Reiter R, Schlabritz-Loutsevitch N, et al. Melatonin membrane receptors in peripheral tissues: distribution and functions. Mol Cell Endocrinol. 2012; 351(2):152-166.

7. Tosini G, Owino S, Guillame J-L, et al. Melatonin receptors: latest insights from mouse models. BioEssays. 2014; 36(8):778-787.

8. Liu J, Clough SJ. Hutchinson AJ, et al. MT1 and MT2 Melatonin Receptors: A Therapeutic Perspective. Annu Rev Pharmacol Toxicol. 2016; 56:361-383.

9. Becker-Andre $M$, Wiesenberg I, Schaeren-Wiemers $N$, et al. Pineal gland hormone melatonin binds and activates an orphan of the nuclear receptor superfamily. J Biol Chem. 1994; 269:28531-28534.

10. Pandi-Perumal S, Trakht I, Srinivasan V, et al. Physiological effects of melatonin: Role of melatonin receptors and signal transduction pathways. Prog Neurobiol. 2008; 85:335-353.

11. Niles $L P$, Armstrong KJ, Castro $L M$, et al. Neural stem cells express melatonin receptors and neurotrophic factors: colocalization of the MT 1 receptor with neuronal and glial markers. BMC neurosci. 2004; 5(1):41.

12. Kong X, Li X, Cai Z, et al. Melatonin regulates the viability and differentiation of rat midbrain neural stem cells. Cell Mol Neurobiol. 2008; 28(4):569-579.

13. Sotthibundhu A, Phansuwan-Pujito $P$, Govitrapong $P$. Melatonin increases proliferation of cultured neural stem cells obtained from adult mouse subventricular zone. J Pineal Res. 2010; 49(3):291-300.

14. Fu J, Zhao S, Liu H, et al. Melatonin promotes proliferation and differentiation of neural stem cells subjected to hypoxia in vitro. J Pineal Res. 2011; 51(1):104-112.

15. Shuai $Y$, Liao L, Su X, et al. Melatonin Treatment Improves Mesenchymal Stem Cells Therapy by Preserving Stemness during Long-term In Vitro Expansion. Theranostics. 2016; 6(11):1899-1917.

16. Mias C, Trouche E, Seguelas M, et al. Ex vivo pretreatment with melatonin improves survival, proangiogenic/mitogenic activity, and efficiency of mesenchymal stem cells injected into ischemic kidney. Stem cells. 2008; 26(7):1749-1757.

17. Zaminy A, Ragerdi Kashani I, Barbarestani $M$, et al. Osteogenic differentiation of rat mesenchymal stem cells from adipose tissue in comparison with bone marrow mesenchymal stem cells: melatonin as a differentiation factor. Iranian Biomed J. 2008; 12(3):133-141.

18. Lee $S$, Jung $Y$, Oh S, et al. Melatonin enhances the human mesenchymal stem cells motility via melatonin receptor 2 coupling with Gaq in skin wound healing. J Pineal Res. 2014; 57(4):393-407.

19. Wu H, Song C, Zhang J, et al. Melatonin-mediated upregulation of GLUT1 blocks exit from pluripotency by increasing the uptake of oxidized vitamin C in mouse embryonic stem cells. FASEB J. 2017; 31(4):1731-1743.

20. Shu T, Fan L, Wu T, et al. Melatonin promotes neuroprotection of induced pluripotent stem cells-derived neural stem cells subjected to $\mathrm{H} 2 \mathrm{O} 2$-induced injury in vitro. Eur J Pharmacol. 2018; 825:143-150.

21. Cho YA, Noh K, Jue SS, et al. Melatonin promotes hepatic differentiation of human dental pulp stem cells: clinical implications for the prevention of liver fibrosis. J Pineal Res. 2015; 58(1):127-135.

22. Majidinia M, Reiter RJ, Shakouri SK, et al. The multiple functions of melatonin in regenerative medicine. Ageing Res Rev. 2018. Available from: $h t t p s: / / d o i . o r g / 10.1016 / j$. arr.2018.04.003.

23. Luchetti F, Canonico B, Bartolini D, et al. Melatonin regulates mesenchymal stem cell differentiation: a review. J Pineal Res. 2014; 56(4):382-397.

24. Nedergaard J, Bengtsson T, Cannon B. Unexpected evidence for active brown adipose tissue in adult humans. Am J Physiol Endocrinol Metab. 2007; 293(2):E444-E452.

25. Saito M, Okamatsu-Ogura $Y$, Matsushita $M$, et al. High incidence of metabolically active brown adipose tissue in healthy adult humans: effects of cold exposure and adiposity. Diabetes. 2009; 58(7):1526-1531.

26. Townsend K, Tseng YH. Brown adipose tissue: recent insights into development, metabolic function and therapeutic potential. Adipocyte. $2012 ; 1$ (1):13-24.

27. Roman S, Agil A, Peran M, et al. Brown adipose tissue and novel therapeutic approaches to treat metabolic disorders. Transl Res. 2015; 165(4):464-479.

28. Wang $Q$, Zhang $M$, Ning $G$, et al. Brown adipose tissue in humans is activated by elevated plasma catecholamines levels and is inversely related to central obesity. PloS one. $2011 ; \mathbf{6}(\mathbf{6}): 21006$.

29. Vosselman MJ, Van der Lans AA, Brans B, et al. Systemic $\beta$-adrenergic stimulation of thermogenesis is not accompanied by brown adipose tissue activity in humans. Diabetes. 2012; 61(12):3106-3113.

30. Boström P, Wu J, Jedrychowski MP, et al. A PGC1-a-dependent myokine that drives brown-fat-like development of white fat and thermogenesis. Nature. 2012 481(7382):463-468.

31. Moreno-Navarrete JM, Ortega F, Serrano $M$, et al. Irisin is expressed and produced by human muscle and adipose tissue in association with obesity and insulin resistance. J Clin Endocrinol Metab. 2013; 98(4):E769-E778.

32. Bartness TJ, Demas GE, Song CK. Seasonal changes in adiposity: the roles of the photoperiod, melatonin and other hormones, and sympathetic nervous system. Exp Biol Med. 2002; 227(6):363-376. 
33. Brydon L, Petit L, Delagrange P, et al. Functional expression of MT2 (Mel1b) melatonin receptors in human PAZ6 adipocytes. Endocrinology. 2001; 142(10):42644271.

34. Alonso-Vale MI, Anhê GF, das Neves Borges-Silva C, et al. Pinealectomy alters adipose tissue adaptability to fasting in rats. Metabolism. 2004 ; 53(4):500-506.

35. Elabd C, Chiellini C, Carmona M, et al. Human Multipotent Adipose-Derived Stem Cells Differentiate into Functional Brown Adipocytes. Stem cells. 2009; 27(11): 2753-2760.

36. Ahfeldt T, Schinzel RT, Lee YK, et al. Programming human pluripotent stem cells into white and brown adipocytes. Nature cell biology. 2012; 14(2):209-219.

37. Nishio $M$, Yoneshiro $T$, Nakahara $M$, et al. Production of functional classical brown adipocytes from human pluripotent stem cells using specific hemopoietin cocktail without gene transfer. Cell metabolism. 2012; 16(3):394-406.

38. Tan DX, Manchester LC, Fuentes-Broto L, et al. Significance and application of melatonin in the regulation of brown adipose tissue metabolism: relation to human obesity. Obes Rev. 2011; 12(3):167-188.

39. Rieck B, Schlaak S. In vivo tracking of rat preadipocytes after autologous transplantation. Ann Plast Surg. 2003; 51(3):294-300.

40. Tseng YH, Kokkotou E, Schulz TJ, et al. New role of bone morphogenetic protein 7 in brown adipogenesis and energy expenditure. Nature. 2008; 454(7207):10001004.

41. Tanzi MC, Farè S. Adipose tissue engineering: state of the art, recent advances and innovative approaches. Expert Rev Med Devices. 2009 ; 6(5):533-551.

42. Itoi $Y$, Takatori $M$, Hyakusoku $\mathrm{H}$, et al. Comparison of readily available scaffolds for adipose tissue engineering using adipose-derived stem cells. JPRAS. 2010; 63(5):858-864.

43. Bonnefont-Rousselot D, Collin F. Melatonin: action as antioxidant and potential applications in human disease and aging. Toxicology. 2010; 278(1):55-67.

44. Reiter RJ, Tan DX, Herman TS, et al. Melatonin as a radioprotective agent: a review. Int J Radiat Oncol Biol Phys. 2004; 59(3):639-653.

45. Le Gouic S, Atgié C, Viguerie-Bascands N, et al. Characterization of a melatonin binding site in Siberian hamster brown adipose tissue. Eur J Pharmacol. 1997; 339(2-3):271-278.

46. Prunet-Marcassus B, Ambid L, Viguerie-Bascands $N$, et al. Evidence for a direct effect of melatonin on mitochondrial genome expression of Siberian hamster brown adipocytes. J Pineal Res. 2001; 30(2):108-115.

47. Kato H, Tanaka G, Masuda S, et al. Melatonin promotes adipogenesis and mitochondrial biogenesis in 3T3-L1 preadipocytes. J Pineal Res. 2015; 59(2):267-275.

48. Rhee $\mathrm{YH}$, Ahn JC. Melatonin attenuated adipogenesis through reduction of the CCAAT/enhancer binding protein beta by regulating the glycogen synthase 3 beta in human mesenchymal stem cells. J Physiol Biochem. 2016; 72(2):145-155.

49. Zhang $L, S u P, X u C$, et al. Melatonin inhibits adipogenesis and enhances osteogenesis of human mesenchymal stem cells by suppressing PPARY expression and enhancing Runx2 expression. J Pineal Res. 2010; 49(4):364-372.

50. Alonso-Vale M. IC, Peres SB, Vernochet $C$, et al. Adipocyte differentiation is inhibited by melatonin through the regulation of C/EBP $\beta$ transcriptional activity. J Pineal Res. 2009; 47(3):221-227.

51. Laitinen JT, Castren E, Vakkuri O, et al. Diurnal rhythm of melatonin binding in the rat suprachiasmatic nucleus. Endocrinology. $1989 ; 124(3): 1585-1587$.

52. Acuña-Castroviejo D, Reiter RJ, Menendez-Pelaez A, et al. Characterization of high-affinity melatonin binding sites in purified cell nuclei of rat liver. J Pineal Res. 1994; 16(2):100-112.

53. Dallmann R, Brown SA, Gachon F. Chronopharmacology: new insights and therapeutic implications. Annu Rev Pharmacol Toxicol. 2014; 54:339-361.

54. McKenna H, van der Horst GT, Reiss I, et al. Clinical chronobiology: a timely consideration in critical care medicine. Crit Care. $2018 ; 22(1): 124$.

55. Dyar KA, Eckel-Mahan KL. Circadian Metabolomics in Time and Space. Front Neurosci. 2017; 11:369.

56. Yang F, Zhang L, Zhu W. Adaptive thermogenesis of brown adipose tissue in tree shrews (Tupaia belangeri): Role of melatonin. J Zool Biosci Res. $2017 ; \mathbf{1}(4): 1-7$.

57. Gao AW, Houtkooper RH. Mitochondrial fission: firing up mitochondria in brown adipose. EMBO J. 2014; 33(5):401-402.

58. Contreras C, Nogueiras R, Diéguez C, et al. Hypothalamus and thermogenesis: heating the BAT, browning the WAT. Mol Cell Endocrinol. 2016; 438:107-115.

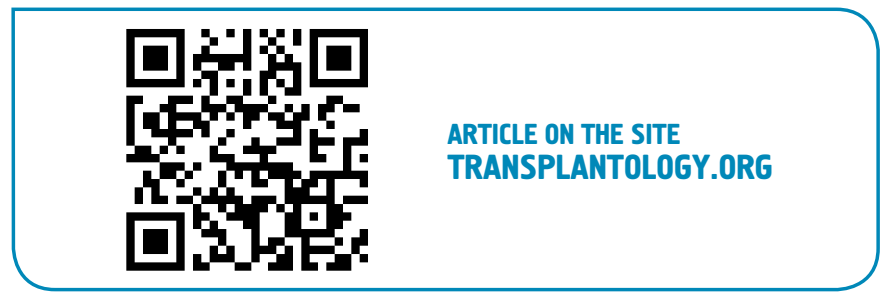

The authors indicate no potential conflicts of interest. 